\title{
Microsatellite Marker for Homozygosity Testing of Putative Doubled Haploids and Characterization of Mimulus Species Derived by a Cross-genera Approach
}

\author{
Jana Murovec, Natasa Stajner, Jernej Jakse, and Branka Javornik ${ }^{1}$ \\ University of Ljubljana, Biotechnical Faculty, Centre for Plant Biotechnology and Breeding, \\ Jamnikarjeva 101, 1000 Ljubljana, Slovenia
}

\begin{abstract}
Additional index words. Mimulus aurantiacus, monkey flower, ovule culture, ornamental plant, degenerative primer, molecular marker

Abstract. A codominant marker for homozygosity testing and species discrimination needed in breeding programs was developed and applied to different Mimulus $L$. species and cultivars. Degenerative primers used to amplify intron 10 of topoisomerase 6 subunit B (top6B) in distant species also amplified the locus in all analyzed Mimulus species. The sequences obtained revealed the presence of a microsatellite motif and were used to design a specific microsatellite primer pair, Mim-top6B, for Mimulus species. The microsatellite marker showed a high degree of polymorphism in Mimulus species, and the heterozygous nature of most $M$. aurantiacus Curtis cultivars. The marker was further used to analyze putative doubled haploids of $M$. aurantiacus and showed that all but one was heterozygous, indicating their hybrid origin.
\end{abstract}

Bush monkey flower (Mimulus aurantiacus) is an important horticultural plant native to North America. To meet market demands for new superior varieties, producers are extending their breeding programs of $M$. aurantiacus, introducing modern biotechnological approaches, such as doubled haploid (DH) production, which already have wide application in various breeding programs and genetic studies. The establishment of completely homozygous lines of a selected species in one generation without the need of self-pollination for several years enables numerous applications of haploids and DHs, such as the production of homozygous lines, induced mutations, chromosome reduction of polyploid species, gene or quantitative trait loci (QTL) mapping, genomics, and transformation experiments (Kasha and Maluszynski, 2003).

Double haploid approaches combine various in vivo, in vitro, or both stimuli, which induce haploid gametes to undergo sporophytic development, resulting in haploid or diploid regenerants in different ratios. The latter can originate from spontaneous "diploidization" of haploid structures through endoreduplication and nuclear fusion (Kasha et al., 2001) or from diploid heterozygous somatic cells and zygotic embryos. To assess the origin of diploids and to exclude undesired heterozygous regenerants from further genetic experiments and breeding programs, homozygosity testing must be performed. Biochemical and molecular marker systems are becoming increasingly important today for homozygosity testing and they are replacing other techniques, such as self-pollination with subsequent progeny testing and morphological markers. The most frequently used molecular markers for homozygosity testing used to be randomly amplified polymorphic DNAs (Eimert et al., 2003; Yahata et al., 2005a, b), but microsatellite markers are used much more nowadays because of their

Received for publication 31 Jan. 2007. Accepted for publication 31 May 2007. This work was supported by research grants P4-0077 and 331-03-831251 from the Slovenian Research Agency, and by HaploTech Project COOP-CT-2003508210 .

${ }^{1}$ Corresponding author. E-mail: branka.javornik@bf.uni-lj.si. codominant nature and unambiguous results. Analysis of the genetic origin of haploid/diploid regenerants using microsatellite markers has been applied in various fruit crops, including clementine (Citrus clementina Hort. ex Tan.) (Germanà and Chiancone, 2003; Germanà et al., 2005), apple (Malus $\times$ domestica Borkh.) (Höfer et al., 2002; Vanwynsberghe et al., 2005), pear (Pyrus communis L.) (Bouvier et al., 2002), and other crops such as wheat (Triticum aestivum L.) (Muranty et al., 2002) and maize (Zea mays L.) (Aulinger et al., 2003; Tang et al., 2006).

To evaluate the results of our haploid induction experiments conducted during the past 3 years, a codominant marker was needed. For homozygosity testing of putative DH plants produced, we first tested some published microsatellites for M. guttatus DC (Awadalla and Ritland, 1997; Kelly and Willis, 1998). Because we could not get any amplification products with $M$. aurantiacus cultivars or other species tested ( $M$. guttatus and M. luteus L.), we tried to amplify intron 10 of topoisomerase 6 subunit B (top $6 B)$, as an alternative marker. Our results confirmed the previously reported cross-species applicability of the top $6 B$ locus, because successful amplification of this locus with degenerative primers in all tested Mimulus species and cultivars was achieved.

The objectives of our study were 1) to develop a simple codominant marker for homozygosity testing of putative DH plants of $M$. aurantiacus, 2) to examine the applicability of the marker for determination of different Mimulus species, and 3) to determine the origin of diploid regenerants from haploid induction experiments.

\section{Materials and Methods}

Plant materials. Six species of the genus Mimulus (M. aurantiacus, M. guttatus, M. luteus, M. primuloides Benth., M. moschatus Lindl., and M. cardinalis Dougl. ex Benth.), of which $M$. aurantiacus was represented by seven accessions ('Champagne', 'Light Yellow', 'Lilac', 'White', 'Wine', line $\mathrm{A}$, and line B), were provided by Flora-Nova Pflanzen $\mathrm{GmbH}$ 
(Hillscheid, Germany). They were grown in the greenhouse of the University of Ljubljana, Biotechnical Faculty, Slovenia for the duration of the growing season.

Forty-four putative DHs were obtained in 2005 after pollination of $M$. aurantiacus 'Lilac' with irradiated (600 Gy) pollen of M. aurantiacus 'White' (J. Murovec and B. Bohanec, unpublished data) and the subsequent rescue of putative gynogenic embryos. The putative $\mathrm{DH}$ plants were grown in vitro at a 16 -h photoperiod.

DNA isolation AND QUANTIFiCATION. Total genomic DNA was extracted from fresh leaves using a modified cetyl trimethylammonium bromide (CTAB) method (Kump and Javornik, 1996). Isolated DNA was resuspended in Tris-EDTA (TE), quantified using a fluorometer (Hoefer DyNA Quant 200; GE Healthcare, Little Chalfont, UK) and diluted to $10 \mathrm{ng} \cdot \mu \mathrm{L}^{-1}$. Samples were stored at $-20{ }^{\circ} \mathrm{C}$.

Preamplification with degenerate primer pair. A degenerate primer pair top6B-rev (GAI GGR TGR TRY TTI GTY TC) and top6B-for (CAR GTK GTI ATI GAR GGW AAY TGG, where $\mathrm{I}$ is inosine, $\mathrm{R}$ is $\mathrm{A} / \mathrm{G}, \mathrm{Y}$ is $\mathrm{C} / \mathrm{T}, \mathrm{K}$ is $\mathrm{G} / \mathrm{T}$, and $\mathrm{W}$ is $\mathrm{A} / \mathrm{T}$ ) had been previously designed from a Humulus lupulus L. genomic sequence (AY588321) to amplify a compound dinucleotide microsatellite repeat $(\mathrm{GT})_{\mathrm{n}}(\mathrm{GA})_{\mathrm{n}}$ residing in the putative intron 10 of gene topoisomerase 6 subunit B (Stajner et al., 2005). The primer pair was used for amplification of a putative microsatellite locus top $6 B$ from $M$. aurantiacus 'Champagne', 'Light Yellow', 'White', 'Lilac', ER19 (progeny from the cross 'Champagne' $\times$ 'Light Yellow'), M. guttatus 'Magic Yellow', and M. luteus.

Amplification of the top $6 B$ locus was performed in a thermal cycler (T-Gradient Thermocycler; Whatman Biometra, Goettingen, Germany) in a volume of $20 \mu \mathrm{L}$ solution containing 100 ng genomic DNA, 1 U Taq-DNA polymerase (Fermentas International, Burlington, $\mathrm{ON}), 1 \times$ supplied polymerase chain reaction (PCR) buffer ( $50 \mathrm{~mm} \mathrm{KCl;} 10 \mathrm{~mm}$ Tris- $\mathrm{HCl}$; $\mathrm{pH}, 8.8)$, $1.5 \mathrm{mM} \mathrm{MgCl}_{2}, 0.2 \mathrm{~mm}$ of each deoxynucleotide triphosphates (dNTP) (Sigma, St. Louis), and $1.5 \mu \mathrm{M}$ of each degenerate primer. A touchdown amplification profile was used, consisting of an initial denaturation step at $94^{\circ} \mathrm{C}$ for $5 \mathrm{~min}$, followed by 10 cycles of denaturation for $45 \mathrm{~s}$ at $94{ }^{\circ} \mathrm{C}$, annealing for $30 \mathrm{~s}$ at $54{ }^{\circ} \mathrm{C}$, with a decrease of $0.4{ }^{\circ} \mathrm{C}$ in every subsequent cycle, and an extension for $1.5 \mathrm{~min}$ at $72^{\circ} \mathrm{C}$. It was followed by 28 cycles of $45 \mathrm{~s}$ at $94{ }^{\circ} \mathrm{C}, 30 \mathrm{~s}$ at $50{ }^{\circ} \mathrm{C}$, and $1.5 \mathrm{~min}$ at $72{ }^{\circ} \mathrm{C}$. A final extension step was carried out at $72{ }^{\circ} \mathrm{C}$ for $8 \mathrm{~min}$. Reaction products were mixed with an equal volume of formamide, heat denatured, and separated on vertical $5 \%$ denaturing polyacrylamide gel in Tris-borate-EDTA (TBE) buffer containing 7.5 M urea. Gels were stained with silver using the Promega Silver Sequence protocol with some modifications (Jakse et al., 2001). Individual DNA fragments were reamplified and the DNA products were controlled by $1.5 \%$ agarose gel electrophoresis for the correct length and quality of reamplified DNA fragment.

Sequence ANALysis. Fragments were sequenced from both sides by the sequencing service (Macrogen, Seoul, Korea) using a DNA analyzer (ABI 3730 XL; Applied Biosystems, Foster City, CA). Sequence trace files were edited and aligned using a CodonCode Alligner (version 1.6.1; CodonCode Corp., Dedham, MA). Homology searches of the sequences were performed by BLASTN, BLASTX, and TBLASTX programs available through the services of the National Center for Biotechnology Information (NCBI) against $\mathrm{nr}$, expressed sequence tags (EST), wgs, and trace databases. The sequences have been submitted to GenBank (accession nos. EF139836, EF139837, EF139838, EF139839, EF139840, EF139841, EF139842, EF139843, and EF139844). Sequences were aligned using ClustalX version 1.81 software (Thompson et al., 1997) and some corrections were done manually around the microsatellite motif region.

Primer design and amplification of microsatellite LOCus. Based on aligned $M$. aurantiacus allele sequences, a Mimulus-specific primer pair Mim-top6B was developed using the PRIMER3 program (Rozen and Skaletsky, 2000). Single nucleotide polymorphism (SNP) positions were avoided to enable complete annealing in the PCR reaction (Fig. 1). The sequence of the primer pair is as follows: $\left(5^{\prime}-3^{\prime}\right)$ Mim-top6Bfor: GGAGCTGGAGGCATAATATC and Mim-top6B-rev: TGGCTGTCATCACTCCTTATTC. Primers were commercially synthesized by MWG Biotech (Ebersberg, Germany), with an 18-bp M13 tail sequence attached to the forward primer for subsequent fluorescent labeling according to Schuelke (2000).

To assess inter- and intraspecific variability, the developed primer pair was tested on individuals from six Mimulus species: M. aurantiacus, M. guttatus, M. luteus, M. cardinalis, $M$. primuloides, and $M$. moschatus, with $M$. aurantiacus represented by seven cultivars. The composition of $10 \mu \mathrm{L}$ PCRs was as follows: $20 \mathrm{ng}$ genomic DNA, $0.25 \mathrm{U}$ Taq-DNA polymerase (Fermentas), $1 \times$ supplied PCR buffer, $2 \mathrm{~mm} \mathrm{MgCl}_{2}, 0.2 \mathrm{~mm}$ of each dNTP (Sigma), 0.2 $\mu \mathrm{M}$ of each unlabeled primer, and $0.075 \mu \mathrm{M}$ of CY5-labeled specific M13 primer $\left(5^{\prime}-3^{\prime}\right.$ : TGTAAAACGACGGCCAGT). The following optimized touchdown protocol was used for PCR after the initial denaturation of $94{ }^{\circ} \mathrm{C}$ for $5 \mathrm{~min}$ : five cycles of $94{ }^{\circ} \mathrm{C}$ for $45 \mathrm{~s}, 60{ }^{\circ} \mathrm{C}$ for $30 \mathrm{~s}$ with a decrease of $-1{ }^{\circ} \mathrm{C}$ per cycle, and $72{ }^{\circ} \mathrm{C}$ for $1.5 \mathrm{~min}$, followed by 25 cycles at the annealing temperature of $55^{\circ} \mathrm{C}$. Reactions were completed by incubating at $72^{\circ} \mathrm{C}$ for $8 \mathrm{~min}$. The amplified products were resolved on $6 \%$ polyacrylamide gel electrophoresis containing $7 \mathrm{~m}$ urea and detected by an automated sequencer (ALFexpress II DNA Analysis System; GE Healthcare). Alleles were precisely sized against a 50 to 500-bp ladder (GE Healthcare) and by the 150-bp internal DNA standard amplified from pBluescriptII plasmid DNA. Results were visualized and analyzed using AlleleLocator software (version 1.03; GE Healthcare).

Genetic ORIGIN OF Regenerants. An optimized simple sequence repeat (SSR) marker protocol was finally applied for homozygosity testing of 44 putative $\mathrm{DH}$ regenerants obtained from haploid induction experiments growing in vitro. The PCR protocol performed was the same as described earlier.

\section{Results and Discussion}

Preamplification with degenerate Primer Pair. Degenerate primer pairs have been developed for the amplification of intron 10 of the top $6 B$ gene, and successful amplification has been shown in several distantly related species (Stajner et al., $2005)$. The first testing of amplification of the top $6 B$ locus in $M$. luteus, $M$. guttatus, and cultivars of $M$. aurantiacus using the degenerate primer pair resulted in DNA fragments ranging in size between $350 \mathrm{bp}$ and $500 \mathrm{bp}$. The separation of amplified fragments on silver-stained polyacrylamide gel revealed probable polymorphisms between cultivars and the genetic 


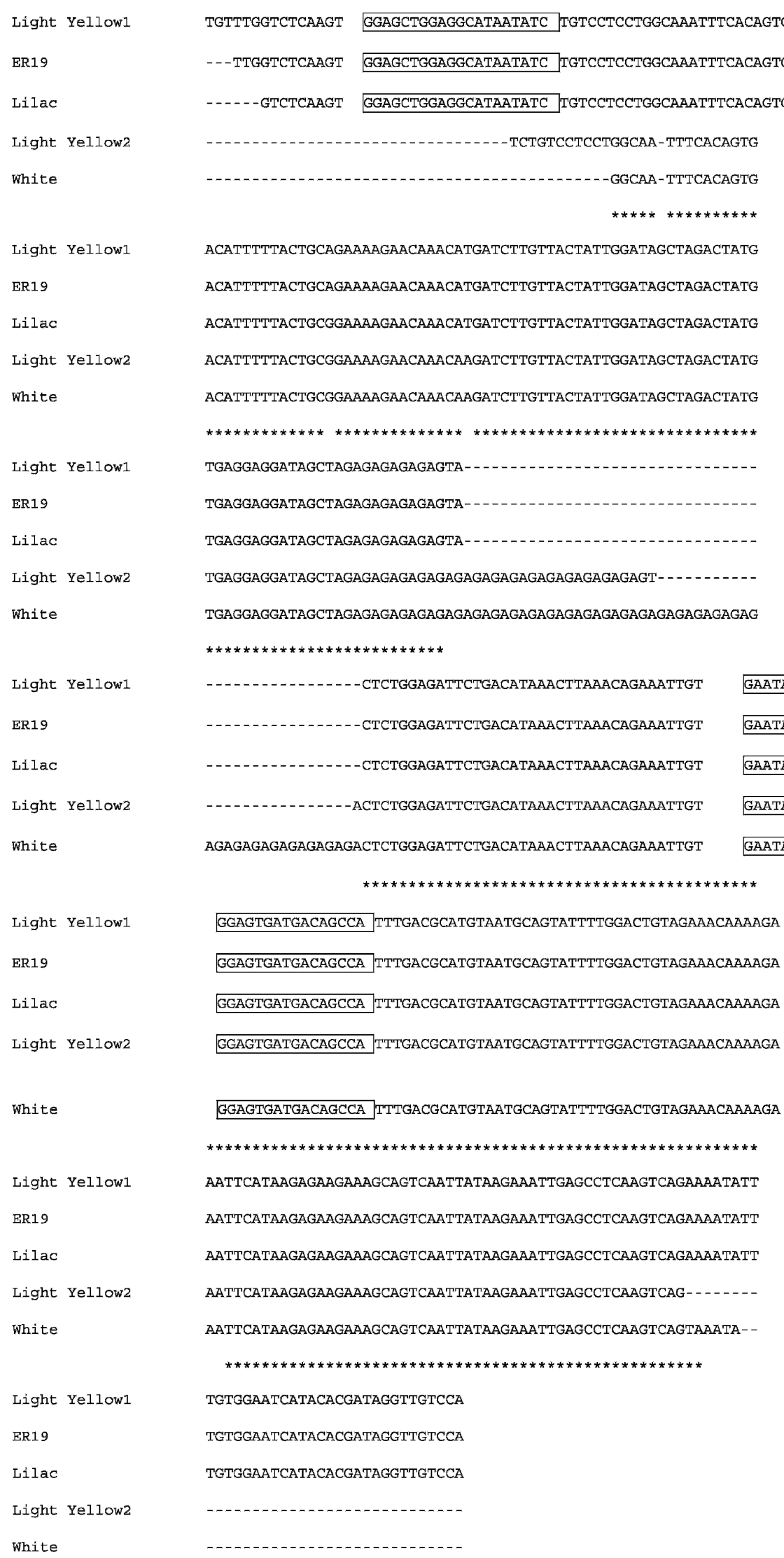

Fig. 1. Multiple sequence alignment of Mimulus aurantiacus 'Light Yellow', ER19, 'Lilac', and 'White' specific DNA fragments amplified with the Mim-top6B primer pair. Dashes represent gaps and asterisks indicate the identity of nucleotides. The primers sequences are in boxes. Missing sequence information at either the $5^{\prime}$ or $3^{\prime}$ end is the result of the sequencing method used and does not represent a deletion or insertion. constitution of cultivars. Mimulus aurantiacus 'Champagne', 'White', 'Light Yellow', and ER19 exhibited a heterozygous nature, whereas M. aurantiacus 'Lilac', M. guttatus, and $M$. luteus were putative homozygotes, with only one fragment at around $350 \mathrm{bp}$. In two samples ('Light Yellow' and ER19), one additional DNA fragment at around 500 bp was amplified (data not shown).

SEquence analysis. Among 13 sequenced fragments, all but two resulted in putative alleles containing a dinucleotide pure microsatellite motif $(\mathrm{AG})_{\mathrm{n}}$ and were therefore used to design a specific microsatellite primer pair (Mim-top6B). The longest amplified bands (around $500 \mathrm{bp}$ ) of M. aurantiacus found in 'Light Yellow' and ER19 (accession nos. EF139836 and EF139839) were confirmed to represent an unspecific amplification, because they did not contain the microsatellite motif and they did not share any sequence homology at the nucleotide or translated level with other sequences. In aligned sequences of specific DNA fragments of M. aurantiacus 'Light Yellow', ER19, 'Lilac', and 'White' (accession nos. EF139837, EF139838, EF139840, EF139841, and EF139842), three SNP positions are revealed (one deletion, one transition, and one transversion) and length polymorphisms are only the result of the variation of dinucleotide repeats (Fig. 1). The variation in the microsatellite repeat number was 6,17 , and $31 \mathrm{AG}$ repeats for the sequenced alleles.

All obtained sequences were searched against NCBI available databases. For all specific sequences amplified in cultivars of M. aurantiacus (accession nos. EF139837, EF139838, EF139840, EF139841, and EF139842), M. guttatus (accession no. EF139844), and M. luteus (accession no. EF139843), significant hits confirmed the similarity with top $6 B$ genes (data not shown). Bands from M. guttatus and M. luteus were identical to each other and to three $M$. guttatus sequences from the trace archive of GenBank (trace identifier nos. 119207748, 1182856220 , and 1223179034), but they shared a lower identity with $M$. aurantiacus sequences at nucleotide or translated amino acid level, at $\approx 90 \%$ (alignment not shown).

To our knowledge, this is the first report of an amplifying microsatellite locus from such diverse genera as Mimulus and Humulus. It is well documented that EST-derived microsatellites can be well conserved among species of the same genus or family (Eujayl et al., 2004; Scott et al., 2000), but not across wider taxonomic levels, such as in our case the orders Rosales (Humulus) and Lamiales (Mimulus). We have thus shown that a microsatellite can be amplified from distantly 
related genera using a degenerate primer approach and that the microsatellite motif is present in the same intron of the same gene, although base repeated units are different. In hop the compound $(\mathrm{GT})_{\mathrm{n}}(\mathrm{GA})_{\mathrm{n}}$ motif is present, whereas in Mimulus only the $(\mathrm{GA})_{\mathrm{n}}$ motif exists.

Primer design and amplification of microsatellite LOCUS. The developed primer pair Mim-top6B was tested on available Mimulus species and cultivars to assess the specificity of locus amplification, and inter- and intraspecific variability. The Mim-top6B locus was amplified in all Mimulus samples (Table 1) and the calculated sizes of the amplified fragments confirmed the allelic polymorphism, because all the differences were multiples of two, which indicates the size variation of microsatellite repeats. Because of the specificity of the newly developed primers, the nonspecific amplification of the longest band in 'Light Yellow' and ER19 was avoided. Two bands were amplified with $M$. aurantiacus 'Champagne', 'Light Yellow', 'White', 'Wine', line A, and line B, whereas only one was amplified with $M$. aurantiacus 'Lilac', M. guttatus 'Magic Yellow', M. luteus, M. cardinalis, M. primuloides, and M. moschatus.

The observed heterozygosity with most $M$. aurantiacus cultivars is valuable for $\mathrm{DH}$ production, because it enables discrimination among regenerants and determination of their origin in early phases of breeding programs. The selection of donor plants heterozygous for the described marker is essential for $\mathrm{DH}$ production involving stimulation of unpollinated female reproductive organs (in vitro culture of flowers, ovaries, ovules, etc.) or male reproductive organs (in vitro culture of microspores or anthers). The regenerated DH should contain only one of two possible alleles of the donor plant, thus proving that the homozygote originated from the spontaneous diploidization of haploid structures, whereas somatic regenerants should inherit both alleles of the donor plant.

In the case of androgenesis, this approach provides sufficient evidence of the haploid origin of diploid regenerants, because there is no other possible explanation for the occurrence of homozygous structures from heterozygous donor plants. In contrast to androgenesis, such a marker in gynogenesis is mostly applicable for the elimination of undesired diploid heterozygotes derived from zygotic embryos or somatic regeneration. Using only one marker, we cannot with certainty confirm a spontaneous DH in the case of difficult elimination of cross- and self-pollination. Fertilization with a sperm cell containing the

Table 1. Amplified microsatellite locus in different Mimulus cultivars with corresponding allele sizes.

\begin{tabular}{ll}
\hline $\begin{array}{l}\text { Evaluated cultivars } \\
\text { of the genus Mimulus }\end{array}$ & $\begin{array}{c}\text { Allele sizes } \\
\text { (bp) }\end{array}$ \\
\hline M. aurantiacus Champagne & 267,249 \\
M. aurantiacus Light Yellow & 235,211 \\
M. aurantiacus Lilac & 211 \\
M. aurantiacus White & 267,249 \\
M. aurantiacus Wine & 233,211 \\
M. aurantiacus line A & 249,211 \\
M. aurantiacus line B & 235,233 \\
M. guttatus Magic Yellow & 221 \\
M. luteus & 221 \\
M. cardinalis & 225 \\
M. primuloides & 229 \\
M. moschatus & 209 \\
\hline
\end{tabular}

same allele as the ovule would result in homozygosity at the analyzed locus, although the rest of the genome would not be homozygous. Further analyses with markers at several loci, or self-pollination with progeny testing, would be needed for final confirmation of a DH. To set up pollination experiments, including pseudo fertilization with irradiated pollen or interspecific pollination, it is also very important to combine genotypes containing different allelic forms.

Differences in allele sizes, as shown in Table 1, between evaluated $M$. aurantiacus cultivars and other Mimulus species, could also be applied for interspecific hybrid confirmations. It could be a reliable alternative when discrimination between true interspecific hybrids and plants derived from spontaneous self-pollination is uncertain using morphological evaluation or comparison of relative genome sizes (Sisko et al., 2003).

Genetic ORIGIN OF REGENERANTS. Several haploid induction experiments have been performed with $M$. aurantiacus in recent years and some haploids have been regenerated in our laboratory. The optimized Mim-top6B SSR marker was applied to verify the origin of the regenerated diploids presumed to be spontaneous DH. The 44 putative DH regenerants were obtained after pseudo fertilization of $M$. aurantiacus 'Lilac' (homozygous allelic profile $211 \mathrm{bp}$ ) with irradiated pollen of M. aurantiacus 'White' (heterozygous allelic profile $267 \mathrm{bp} /$ 249 bp; Table 1).

Forty-three of the evaluated regenerants were heterozygous and only one was homozygous for the marker tested. The heterozygotes exhibit the allele of the mother plant (211 bp) and one of the two alleles of the pollen donor plant (267 bp or 249 $\mathrm{bp}$ ), thus demonstrating their hybrid origin. We can conclude from these results that the heterozygous regenerants resulted from pollination of the egg cells of $M$. aurantiacus 'Lilac' with sperm cells of $M$. aurantiacus 'White.' The homozygous regenerant is probably a spontaneous $\mathrm{DH}$ originating from the egg cell of $M$. aurantiacus 'Lilac', because self-pollination, which would also result in homozygosity of the locus tested, was prevented by emasculation of the pollinated flowers. Somatic regeneration could also be excluded as another possible origin of the homozygous regenerant, because did not observe any in our extensive experiments of inoculated ovules. In addition, the emergence of the regenerating embryos from the ovules morphologically resembled seed germination.

This is a report of an original approach for the establishment of a codominant marker system for homozygosity testing of putative DH plants using cross-genera amplification. It shows the potential application of microsatellite loci located in introns of coding regions as universal markers for cross-species amplifications. The advantage of this approach is the avoidance of microsatellite isolation for each species, which reduces the time and funds needed for the development of a reliable and unambiguous molecular marker system.

The degenerate primer pair successfully amplified intron 10 of the top $6 B$ gene, revealing the polymorphism of this locus in Mimulus species and the heterozygosity of most $M$. aurantiacus cultivars. This agrees with previous results of the successful amplification of this region in H. lupulus, H. japonicus Sieb. \& Zucc., Cannabis sativa L., Medicago sativa L., Urtica dioica L., Helianthus annuus L., and 30 different species of Trifolium L. reported by Stajner et al. (2005). Sequence analysis of the amplified fragments confirmed assumptions concerning the presence of microsatellite sequences in the investigated region, and supplements previously reported results. 
In conclusion, the results of our study demonstrate that this microsatellite locus is a valuable marker for homozygosity testing of $M$. aurantiacus, because it allows efficient selection of donor plants and determination of regenerants at a very early stage of the work program (at the in vitro tissue culture phase) to small amounts of starting material, thus saving labor and costs in the laboratory and subsequently in the greenhouse. The amplification of this microsatellite in other Mimulus species is another important contribution, because it can be used for interspecific hybrid determination as a result of the amplification of different alleles at the same locus in those species. The approach using top $6 B$ degenerative primers could also be applied in DH confirmations of other species, because wide cross-species amplification has been demonstrated.

\section{Literature Cited}

Aulinger, I.E., S.O. Peter, J.E. Schmid, and P. Stamp. 2003. Rapid attainment of a doubled haploid line from transgenic maize (Zea mays L.) plants by means of anther culture. In Vitro Cell. Dev. Biol. Plant 39:165-170.

Awadalla, P. and K. Ritland. 1997. Microsatellite variation and evolution in the Mimulus guttatus species complex with contrasting mating systems. Mol. Biol. Evol. 14:1023-1034.

Bouvier, L., P. Guérif, M. Djulbic, C.E. Durel, E. Chevreau, and Y. Lespinasse. 2002. Chromosome doubling of pear haploid plants and homozygosity assessment using isozyme and microsatellite markers. Euphytica 123:255-262.

Eimert, K., G. Reutter, and B. Strolka. 2003. Fast and reliable detection of doubled-haploids in Asparagus officinalis by stringent RAPDPCR. J. Agr. Sci. 141:73-78.

Eujayl, I., M.K. Sledge, L. Wang, G.D. May, K. Chekhovskiy, J.C. Zwonitzer, and M.A.R. Milan. 2004. Medicago truncatula ESTSSRs reveal cross-species genetic markers for Medicago spp. Theor. Appl. Genet. 108:414-422.

Germanà, M.A. and B. Chiancone. 2003. Improvement of Citrus clementina Hort. Ex Tan. microspore-derived embryoid induction and regeneration. Plant Cell Rpt. 22:181-187.

Germanà, M.A., B. Chiancone, O. Lain, and R. Testolin. 2005. Anther culture in Citrus clementina: A way to regenerate tri-haploids. Austral. J. Agr. Res. 56:839-845.

Höfer, M., A. Gomez, E. Aguiriano, J.A. Manzanera, and M.A. Bueno. 2002. Analysis of simple sequence repeat markers in homozygous lines of apple. Plant Breed. 121:159-162.

Jakse, J., K. Kindlhofer, and B. Javornik. 2001. Assessment of genetic variation and differentiation of hop genotypes by microsatellite and AFLP markers. Genome 44:773-782.

Kasha, K.J., T.C. Hu, R. Oro, E. Simion, and Y.S. Shim. 2001. Nuclear fusion leads to chromosome doubling during mannitol pretreatment of barley (Hordeum vulgare L.) microspores. J. Expt. Bot. 52:1227-1238.
Kasha, K.J. and M. Maluszynski. 2003. Production of doubled haploids in crop plants: An introduction, p. 1-4. In: M. Maluszynski, K.J. Kasha, B.P. Forster, and I. Szarejko (eds.). Doubled haploid production in crop plants: A manual. Kluwer Academic Publishers, Dordrecht, The Netherlands.

Kelly, A.J. and J.H. Willis. 1998. Polymorphic microsatellite loci in Mimulus guttatus and related species. Mol. Ecol. 7:769-774.

Kump, B. and B. Javornik. 1996. Evaluation of genetic variability among common buckwheat (Fagopyrum esculentum Moench) populations by RAPD markers. Plant Sci. 114:149-158.

Muranty, H., P. Sourdille, S. Bernard, and M. Bernard. 2002. Genetic characterization of spontaneous diploid androgenetic wheat and triticale plants. Plant Breed. 121:470-474.

Rozen, S. and H.J. Skaletsky. 2000. Primer3 on WWW for general users and for biologist programmers, p. 365-386. In: S. Krawetz and S. Misener (eds.). Bioinformatics methods and protocols: Methods in molecular biology. Humana Press, Totowa, NJ.

Schuelke, M. 2000. An economic method for the fluorescent labeling of PCR fragments. Nat. Biotechnol. 18:233-234.

Scott, K.D., P. Eggler, G. Seaton, M. Rossetto, E.M. Ablett, L.S. Lee, and R.J. Henry. 2000. Analysis of SSRs derived from grape ESTs. Theor. Appl. Genet. 100:697-712.

Sisko, M., A. Ivancic, and B. Bohanec. 2003. Genome size analysis in the genus Cucurbita and its use for determination of interspecific hybrids obtained using the embryo-rescue technique. Plant Sci. 165:663-669.

Stajner, N., J. Jakse, P. Kozjak, and B. Javornik. 2005. The isolation and characterization of microsatellites in hop (Humulus lupulus L.). Plant Sci. 168:213-221.

Tang, F., Y. Tao, T. Zhao, and G. Wang. 2006. In vitro production of haploid and doubled haploid plants from pollinated ovaries of maize (Zea mays L.). Plant Cell Tissue Organ Cult. 84:233-237.

Thompson, J.D., T.J. Gibson, F. Plewniak, F. Jeanmougin, and D.G. Higgins. 1997. The Clustal_X windows interface: Flexible strategies for multiple sequence alignment aided by quality analysis tools. Nucl. Acids Res. 25:4876-4882.

Vanwynsberghe, L., K. De Witte, E. Coart, and J. Keulemans. 2005. Limited application of homozygous genotypes in apple breeding. Plant Breed. 124:399-403.

Yahata, M., S. Harusaki, H. Komatsu, K. Takami, H. Kunitake, T. Yabuya, K. Yamashita, and P. Toolapong. 2005a. Morphological characterization and molecular verification of a fertile haploid pummelo (Citrus grandis Osbeck). J. Amer. Soc. Hort. Sci. 130:34-40.

Yahata, M., H. Kunitake, T. Yabuya, K. Yamashita, Y. Kashihara, and H. Komatsu. 2005b. Production of a doubled haploid from a haploid pummelo using colchicine treatment of axillary shoot buds. J. Amer. Soc. Hort. Sci. 130:899-903. 